\title{
UPLC-MS/MS Method for the Determination of Hyperoside and Application to Pharmacokinetics Study in Rat After Different Administration Routes
}

\author{
Wenjing Yuan ${ }^{1,2,3} \cdot$ Jingjing Wang ${ }^{1,2,3} \cdot$ Xiaofei An ${ }^{6} \cdot$ Mingxin Dai ${ }^{1,2,3} \cdot$ Zhenzhou Jiang $^{1,2,3} \cdot$ Luyong Zhang $^{1,4}$. \\ Sen $\mathrm{Yu}^{5} \cdot$ Xin Huang ${ }^{1,2,3,7}$ (D)
}

Received: 5 November 2020 / Revised: 8 December 2020 / Accepted: 30 December 2020 / Published online: 15 January 2021

(c) The Author(s), under exclusive licence to Springer-Verlag GmbH, DE part of Springer Nature 2021

\begin{abstract}
A rapid and sensitive UPLC-MS/MS method was developed and fully validated for the quantification of hyperoside in rat plasma after intragastric, intraperitoneal and intravenous administration. Geniposide was used as an internal standard, and simple liquid-liquid extraction by ethyl acetate was utilized for to extracting the analytes from the rat plasma samples. Chromatographic separation was carried out on an InfinityLab Poroshell 120EC-C18column $(2.1 \mathrm{~mm} \times 50 \mathrm{~mm}, 1.9$-Micro, Agilent technologies, USA). The mobile phase consisted of methanol (A) and water (B) (containing $0.1 \%$ acetic acid) at a flow rate of $0.4 \mathrm{~mL} / \mathrm{min}$. A run time of $3 \mathrm{~min}$ for each sample made it possible to analyze more than 300 plasma samples per day. The validated linear ranges of hyperoside were $2-1000 \mathrm{ng} / \mathrm{mL}$ in rat plasma. The intra-day and inter-day precision were within $2.6-9.3 \%$, and accuracy were $\pm 8.6 \%$. And the results of recovery and matrix interference studies were well within the accepted variability limits. Finally, this method was fully validated and successfully applied to the pharmacokinetic studies of hyperoside via different administration routes in rats.
\end{abstract}

Keywords Hyperoside $\cdot$ UPLC-MS/MS $\cdot$ Pharmacokinetic study $\cdot$ Different administration routes

$\begin{array}{ll}\text { Abbreviations } \\ \text { Hyp } & \text { Hyperoside } \\ \text { IS } & \text { Internal standard } \\ \text { LLOQ } & \text { Lower limit of quantification } \\ \text { QC } & \text { Quality control } \\ \text { ESI } & \text { Electrospray ionization } \\ \text { CE } & \text { Collision energy values } \\ \text { TEM } & \text { Temperature } \\ \text { HL } & \text { Half life }\end{array}$

Sen $\mathrm{Yu}$

sen.yu@mosim.com

$\triangle$ Xin Huang

huangxin@cpu.edu.cn

1 Institute of Pharmaceutical Research, New Drug Screening Center, China Pharmaceutical University, Nanjing 210009, People's Republic of China

2 Jiangsu Center for Pharmacodynamics Research and Evaluation, China Pharmaceutical University, Nanjing 210009, People's Republic of China

3 Key Laboratory of Drug Quality Control and Pharmacovigilance, Ministry of Education,
AUC Area under curve

MRT Mean residence time

\section{Introduction}

Hyperoside (quercetin 3-O-galactoside, Hyp) is one of the flavonol glycosides. It was detected in many plant species, such as Abelmoschus manihot [1], Apocynum venetum $L$. [2], Crataegus pinnatifida [3], etc. The ethanol extract of the

China Pharmaceutical University, Nanjing 210009, People's Republic of China

4 Center for Drug Screening and Pharmacodynamics Evaluation, School of Pharmacy, Guangdong Pharmaceutical University, Guangzhou 510006, People's Republic of China

5 Mosim Co., Ltd, Nanjing 210009, People's Republic of China

6 Jiangsu Province Hospital of Chinese Medicine, Affiliated Hospital of Nanjing University of Chinese Medicine, Nanjing 210009, People's Republic of China

7 National Nanjing Center for Drug Screening, China Pharmaceutical University, Nanjing 210009, People's Republic of China 
flower in Abelmoschus manihot is made as Huangkui capsule and has been used to treat patients with kidney diseases [4]. The extracts of Apocynum venetum $L$. exerted an protective effect on pirarubicin-induced cardiotoxicity [5]. Zhang [6] reported that hawthorn extracts exhibited antibacterial activity. So hyperoside may be a potential medicinal ingredient. At the same time, it is reported that hyperoside has extensive pharmacological effects. For examples, it could induce both autophagy and apoptosis of cancer cells [7]. In addition, hyperoside was reported to have anti-inflammatory and antioxidant activities [8]. While protecting the cardiomyocytes [9], cortical neurons [10] and renal [11] from ischemia/reperfusion injury, it can also enhance immunity [12], protect the liver [13] and ameliorate depression [14]. A recent study has been reported hyperoside was one of the key molecules of Qing-Fei-Pai-Du decoction in the treatment of COVID-19 [15]. Based on the researches described above, hyperoside has good prospects for medicine.

To further understand its pharmacological mechanism in vivo, it is necessary to explore its pharmacokinetics. Ai et al. [16] studied the pharmacokinetic changes of hyperoside in vivo by gavage and intravenous injection. Both Cmax and AUC were positively correlated with the dose, and the correlation coefficients were 0.998 and 0.999 , respectively, indicating that the elimination of hyperoside was in line with the characteristics of linear kinetics. The bioavailability of hyperoside was low, with an oral bioavailability of only $1 \%$, and pre-administration of verapamil increased its bioavailability by $2.4 \%$ [17]. The low bioavailability of hyperoside may affect its distribution in various tissues and even its efficacy. In addition, some scholars have studied the possibility of hyperoside and its metabolites to reach the nervous system and the pharmacokinetics in the brain based on the protective effect of hyperoside on the nervous system. It was found that the content of hyperoside and its metabolites in the brain was not detected after intragastric administration, but it could be detected after intraperitoneal injection [18]. Therefore, different routes of administration may affect its distribution in tissues, thus affecting its efficacy. For examples, Ganlixin has an obvious clinical effect in the treatment of chronic toxic hepatitis, but has poor absorption in oral preparations. Pharmacokinetic studies show its absolute bioavailability is only about $3 \%$, so Ganlixin is well suited for injection [19]. Besides, the pharmacokinetics of Topotecan in different routes were studied. Plasma concentrations at elimination phase following subcutaneous administration were much higher than oral administration and intravenous administration [20]. In the study of pharmacokinetics of puerarin (the principal component of Tongqiao Sanyu Prescription) in different administration, the bioavailability of nasal administration was higher than that of oral administration, which can provide some scientific foundation for the method of administration and the dosage form of Tongqiao Sanyu prescription [21]. Intraperitoneal injection [11, 22, 23] was mostly used in the studies on the efficacy of hyperoside, while gavage was always used in a long-time study [24, 25]. Whether the physiological basis of hyperoside is related to the amount of hyperoside exposed in vivo, there has no reports of comparing different administration routes. Therefore, it is necessary to investigate the dynamic changes of hyperoside under different administration routes, and obtain its pharmacokinetic parameters, so that the method of administration, dosage, interval and course of treatment can be determined scientifically, which can improve clinical treatment level.

Several analytical methods have been reported for the determination of hyperoside in biological samples. Ai et al. [16] reported that when administered by gavage, hyperoside could not be detected in serum by HPLC. Therefore, they adopted the method of hydrolysis by $\beta$-glucoside acid enzyme, measuring the concentration of quercetin after hydrolysis, and then the conversion to the content of hyperoside, and the lower limit of detection was $0.01 \mu \mathrm{g} / \mathrm{mL}$. However, quercetin is a metabolite of hyperoside after desaccharification [26], so the above determination method is questionable. The concentration of hyperoside in plasma was also determined via HPLC by Liu [27]. But, the lower limit of quantification (LLOQ) was just $0.892 \mu \mathrm{g} / \mathrm{mL}$. Besides, Yin et al. [28] determined hyperoside and isoquercitrin in rat plasma by membrane-protected micro-solid-phase extraction with HPLC. The linear range was between 1 and $120 \mu \mathrm{g} / \mathrm{mL}$. The method was not sensitive but expensive. In addition, hyperoside was detected in plasma by UPLC-MS by Tan [29], but it takes $10 \mathrm{~min}$. Yin [26] improved the method of detection of hyperoside in plasma of beagle dogs after the administration of Hypericum perforatum extract by LC-ESI MS/MS, and the analysis time was $2 \mathrm{~min}$. However, the linear range of this method was $10-5000 \mathrm{ng} / \mathrm{mL}$. In summary, the reported methods have the problems of long analysis time, poor sensitivity and high cost. Therefore, it is necessary to establish a sensitive, convenient, simple and low-cost analytical method to fastly determine compounds.

In this paper, we developed a novel UPLC-MS/MS method for the quantification of hyperoside in rat plasma. The method had the advantages of short analysis time, high sensitivity, good reproducibility and simple sample processing. It was applied for the pharmacokinetic study of hyperoside in different administration routes, so as to provide guidance for drug development and clinical rational application.

\section{Materials and Methods}

\section{Chemicals and Reagents}

The reference standards of hyperoside ( $\geq 98 \%$ pure) and geniposide ( $\geq 98 \%$ pure, internal standard) were purchased from Shanghai Yuanye Bio-Technology Co. Ltd. 
(Shanghai, China); their chemical structures are shown in Fig. 1. Methanol of HPLC grade were obtained from Merck KGaA (Darmstadt, Germany). Acetic acid glacialof HPLC grade was obtained from Tedia company (USA). Ultrapure water was purified by an GenPure UV-TOC/UF xCAD plus (Thermo Electrion LED GmbH, Germany). Other reagents and chemicals were of analytical grade.

\section{Ultra Performance Liquid Chromatography-Mass Spectrometry Analysis}

Chromatography was achieved using an Nexera UHPLC System. Chromatographic separation was carried out on an InfinityLab Poroshell 120 EC-C18 column $(2.1 \mathrm{~mm} \times 50 \mathrm{~mm}, 1.9$-Micron, Agilent technologies, USA). The column temperature was maintained at $40{ }^{\circ} \mathrm{C}$. The mobile phase consisted of methanol (A) and water (B) (containing $0.1 \%$ acetic acid glacial). The gradient condition was: 0-0.3 min, linear from 22 to $33 \% \mathrm{~A} ; 0.3-0.7 \mathrm{~min}$, held at $33 \% \mathrm{~A}$ for $0.4 \mathrm{~min}$; $0.71-2.0 \mathrm{~min}$, linear from 70 to $75 \%$ $\mathrm{A}$, and then an immediate reduction to $22 \%$ A for equilibration of the column. The total run time was $3 \mathrm{~min}$. The flow rate was maintained at $0.4 \mathrm{~mL} / \mathrm{min}$, and the injection volume was $3.0 \mu \mathrm{L}$.

The AB SCIEX QTRAP 5500 (AB SCIEX, Framingham, MA, USA) was connected to the UPLC system via an electrospray ionization (ESI) interface. The source operated in negative ion mode at an ion spray voltage of $-4500 \mathrm{~V}$. The collision energy values (CE) and temperature (TEM) were - $30 \mathrm{~V}$ and $450{ }^{\circ} \mathrm{C}$, respectively. The mass spectrometer was operated in multiple reaction monitoring (MRM) of the transitions of $\mathrm{m} / \mathrm{z} 463.0 \rightarrow \mathrm{m} / \mathrm{z} 300.1$ for hyperoside and $\mathrm{m} / \mathrm{z}$ $387.0 \rightarrow m / z 225.1$ for geniposide. Figure 2 shows the fullscan product ion scan spectra and the fragmentation pathways of the hyperoside and geniposide.

\section{Statistical Analyses}

Data acquisition and processing were performed with Analyst 1.6.2 software (Applied Biosystems [AB Sciex]). The

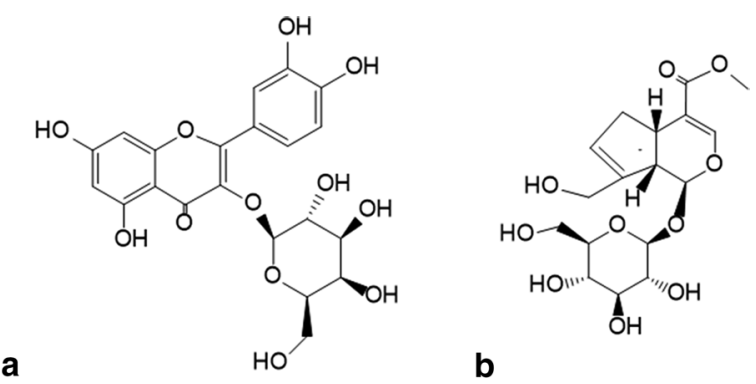

Fig. 1 Chemical structures of hyperoside (a) and geniposide (b) pharmacokinetic parameters were analyzed using Phoenix WinNonlin 7.0 software (Certara, USA).

\section{Preparation of Standards and Quality Control Samples}

Stock solutions of hyperoside and geniposide with concentration of $1.0 \mathrm{mg} / \mathrm{mL}$ were prepared in methanol and stored at $-20{ }^{\circ} \mathrm{C}$. Working solutions were prepared by stock solutions via diluting dilution with methanol to the final concentrations. Nine levels of drug-plasma calibration standards were constructed, with final concentrations of 2, 5, 10, 20, $50,100,200,500,1000 \mathrm{ng} / \mathrm{mL}$, through proper dilution of working solutions and spiking into the blank rat plasma. The QC samples of hyperoside were similarly prepared in blank plasma, to obtain final concentrations of 5, 20, 200 and $800 \mathrm{ng} / \mathrm{mL}$. The QC samples were used to study the precision, accuracy, matrix effect, extraction recovery and stability under different storage conditions.

\section{Plasma Sample Preparation}

Rat plasma $(50 \mu \mathrm{L})$ was added into $1.5 \mathrm{~mL}$ centrifuge tube, then was mixed with $1 \mathrm{~mL}$ ethyl acetate. After the mixtures was vortexed for $3 \mathrm{~min}$, the sample was centrifugated at $15,000 \mathrm{rpm}$ for $10 \mathrm{~min}$. Then, the supernatant was transferred to another $1.5 \mathrm{~mL}$ centrifuge tube and evaporated to dryness at $45^{\circ} \mathrm{C}$ via a vacuum concentration apparatus. The residue was reconstituted in $100 \mu \mathrm{L}$ solution consisting of methanol and water (containing $0.1 \%$ acetic acid glacial, $50: 50, \mathrm{v} / \mathrm{v}$ ), and centrifuged at $15,000 \mathrm{rpm}$ for $10 \mathrm{~min}$. The solution $(3 \mu \mathrm{L})$ was used for analysis.

\section{Application to a Pharmacokinetic Study}

Twenty-one male specific pathogen-free wistar rats (190-200 g) were purchased from Vital River Laboratory Animal Technology (Beijing, China) and were kept in standard laboratory conditions (12 h light/dark cycle, 40-70\% humidity, and a constant temperature of $22-24{ }^{\circ} \mathrm{C}$ ). Animal welfare and experimental procedures were performed in compliance with the National Institutes of Health Guide for the Care and Use of Laboratory Animals and the related ethical regulations of the Institutional Animal Care and Use Committee of China Pharmaceutical University. The rats were randomly divided into three groups $(n=7)$ : Hyperoside was administered to Group $1(100 \mathrm{mg} / \mathrm{kg})$ via gavage, and Group $2(100 \mathrm{mg} / \mathrm{kg})$ via intraperitoneal injection and Group $3(2 \mathrm{mg} / \mathrm{kg})$ via tail intravenous injection. Whole blood samples $(100 \mu \mathrm{L})$ were collected in heparinized polythene tubes at $0.033,0.083,0.166,0.25,0.50,0.75,1,2,4,6,8,10$ and $12 \mathrm{~h}$ after administration of hyperoside, and immediately 
Fig. 2 Full-scan product ion spectra of parent ions and fragmentation pathways for hyperoside (a) and geniposide (b). a Product ion spectrum of [M-H] —of hyperoside at $\mathrm{m} / \mathrm{z}$. 463.0; $\mathrm{b}$ product ion spectrum of [M-H] —of geniposide at $\mathrm{m} / \mathrm{z}$ 387.0
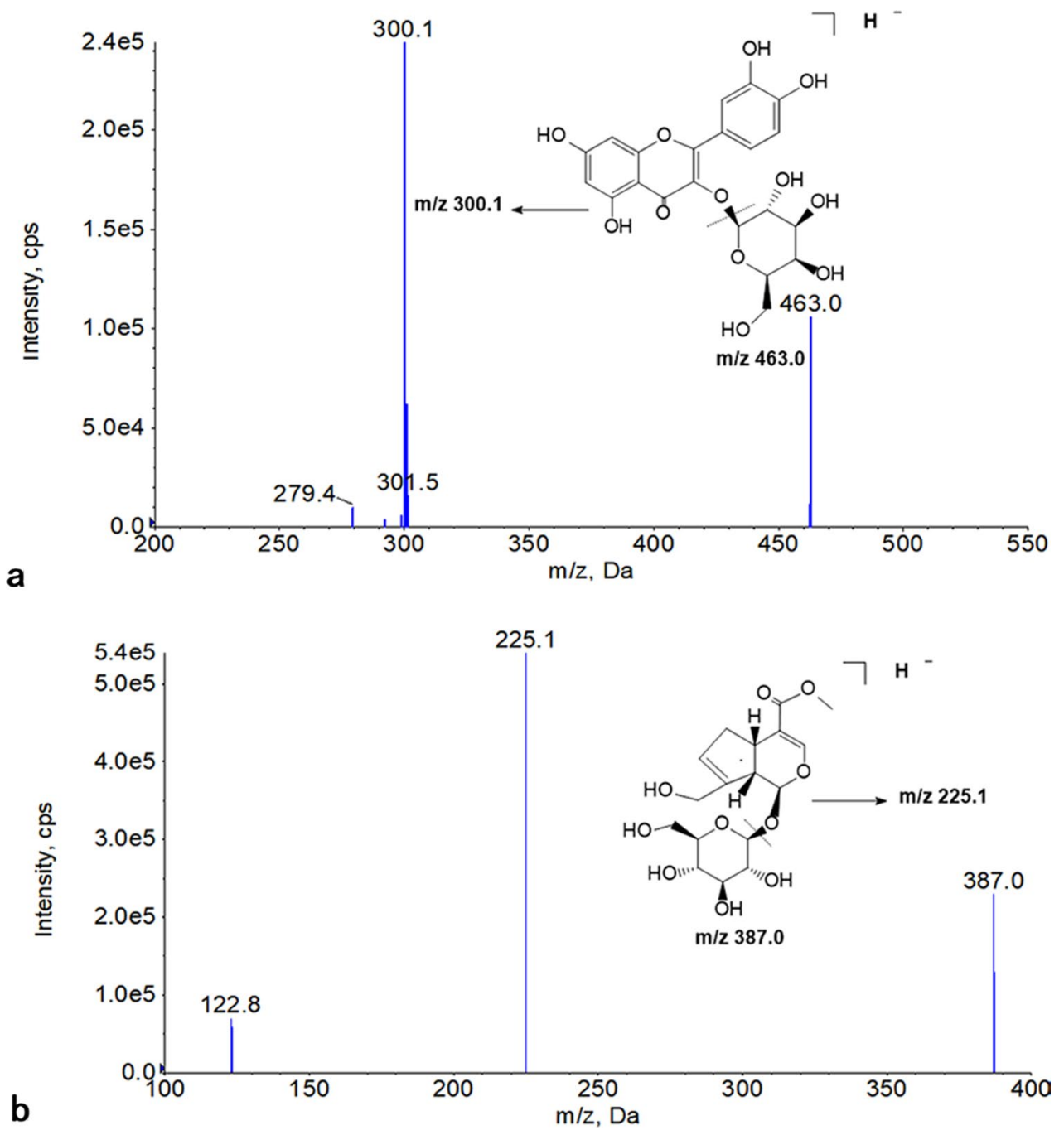

centrifuged at $4000 \mathrm{rpm}$ for $10 \mathrm{~min}$ at $4{ }^{\circ} \mathrm{C}$ to obtain plasma. All samples were stored at $-20{ }^{\circ} \mathrm{C}$ until analyzed.

\section{Results}

The specificity of the method was assessed by comparing the chromatographic profiles of blank plasma obtained from six rats. No visible interfering peaks was observed in the retention time windows of the hyperoside and internal standard (Fig. 3a, b). Hyperoside and IS were separated well, with good peak shapes and resolution. The retention times of hyperoside and internal standard were $1.54 \mathrm{~min}$ and 1.12 min, respectively (Fig. 3c, d). The chromatographic profiles of plasma sample obtained from rat are shown in Fig. 3e, f (e: hyperoside, f: IS).

The calibration curve was constructed by the measured peak area ratios (hyperoside/IS) as the vertical axis and the nominal concentration of hyperoside as the horizontal axis using weighted linear regression $\left(w=1 / x^{2}\right)$ analysis. The linearity was investigated using five calibration standards and the linear range was $2.00-1000 \mathrm{ng} / \mathrm{mL}$ for hyperoside in rat plasma. The correlation coefficient $(r)$ was $>0.99$. The LLOQ values of hyperoside in rat plasma was $2.00 \mathrm{ng} /$ $\mathrm{mL}$. And the accuracies were expressed by relative error $[\mathrm{RE} \%, \mathrm{RE} \%=$ (the measured concentration-the spiked concentration)/the spiked concentration $\times 100]$. As shown in Table 1, the accuracies (RE\%) of hyperoside at the LLOQ was $-8.5 \%$, which met the quantitative detection of analytes. The intra-day and inter-day precision (RSD) were within $2.6-9.3 \%$, and the accuracy (RE\%) were $\pm 8.6 \%$. These results indicated this method can provide acceptable precision, accuracy and reproducibility for determination of hyperoside in rat plasma.

The extraction recoveries of hyperoside were $81.4 \pm 8.9$, $89.7 \pm 5.6,82.9 \pm 3.3,78.2 \pm 4.0 \%$ at $5,20,200$ and $800 \mathrm{ng} /$ $\mathrm{mL}$, respectively. In this method, the recovery rate of extraction at various concentrations is relatively stable. The matrix effect of hyperoside were $92.2 \pm 6.6,101.2 \pm 7.4,100.4 \pm 4.7$, and $91.1 \pm 7.8 \%$ at $5,20,200$ and $800 \mathrm{ng} / \mathrm{mL}$, respectively. 

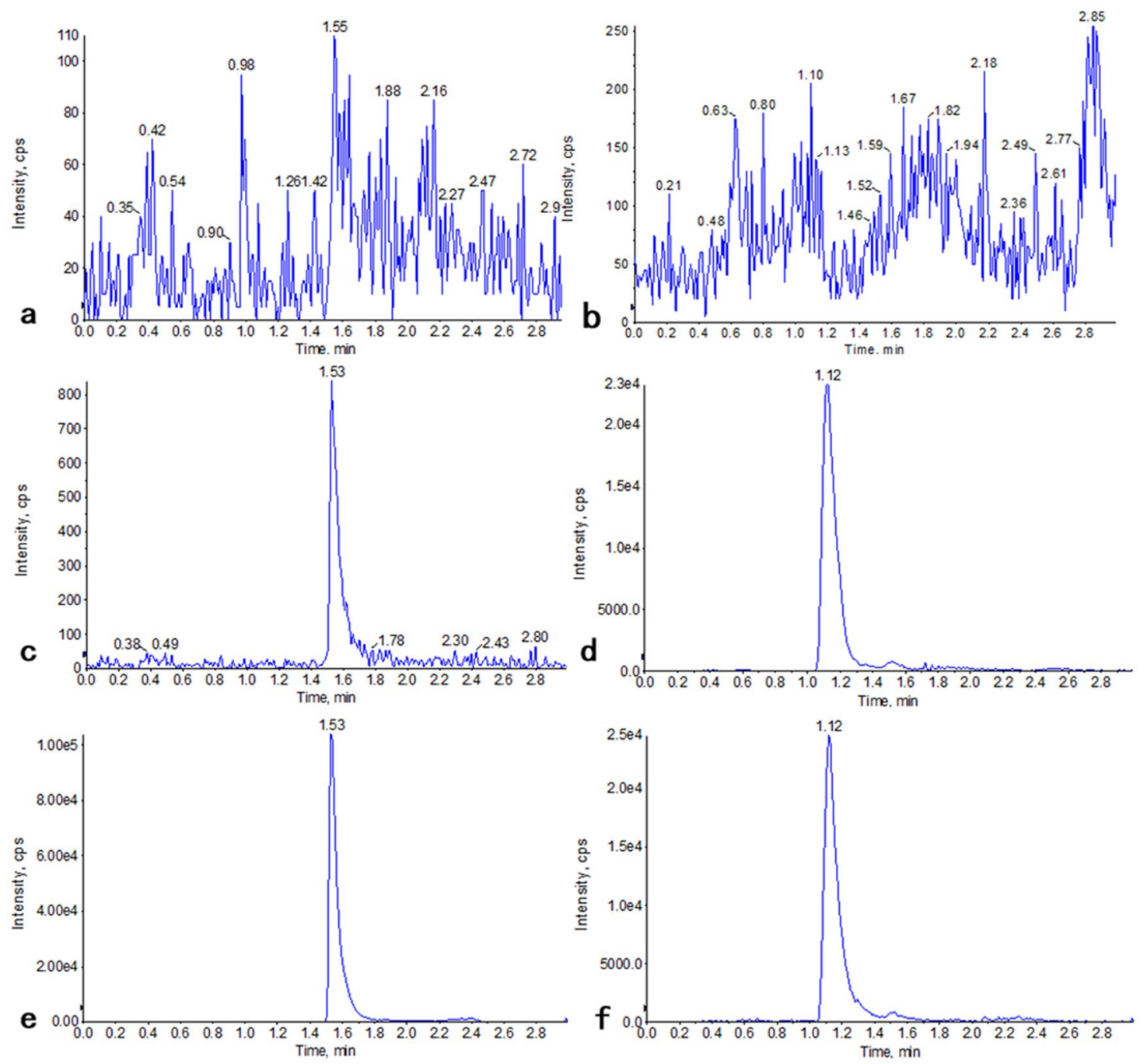

Fig. 3 Typical MRM chromatograms of sample. Double blank sample (a: hyperoside and b: IS); plasma sample spiked with hyperoside (c) at LLOQ and IS (d); plasma sample obtained from rat (e: hyperoside, f: IS)

Table 1 Accuracy and precision for the determination of hyperoside

\begin{tabular}{|c|c|c|c|c|c|c|c|}
\hline \multirow[t]{2}{*}{ Analytes } & \multirow{2}{*}{$\begin{array}{l}\text { Spiked concentra- } \\
\text { tion }(\mathrm{ng} / \mathrm{mL})\end{array}$} & \multicolumn{3}{|l|}{ Intra-day precision $(n=5)$} & \multicolumn{3}{|c|}{ Inter-day precision $(n=15)$} \\
\hline & & Measured $($ mean $\pm S D)$ & $\mathrm{RE}(\%)$ & $\operatorname{RSD}(\%)$ & Measured (mean $\pm \mathrm{SD})$ & $\mathrm{RE}(\%)$ & $\operatorname{RSD}(\%)$ \\
\hline \multirow[t]{4}{*}{ Нyр } & 5 & $5.02 \pm 0.13$ & 0.4 & 2.6 & $5.4 \pm 0.51$ & 8.0 & 9.3 \\
\hline & 20 & $18.88 \pm 0.90$ & -5.6 & 4.8 & $18.9 \pm 0.91$ & -5.5 & 4.8 \\
\hline & 200 & $194.4 \pm 10.1$ & -2.8 & 5.2 & $195 \pm 10.9$ & -2.5 & 5.6 \\
\hline & 800 & $731.4 \pm 25.3$ & -8.6 & 3.5 & $774 \pm 42.6$ & -3.3 & 5.5 \\
\hline
\end{tabular}

These results indicated that the present method was in the absence of a significant matrix effect. In this study, ion enhancement or suppression from rat plasma matrix is negligible.

The stability of hyperoside was evaluated at the four quality control (QC) levels $(5,20,200$, and $800 \mathrm{ng} / \mathrm{mL})$ under different storage conditions in rat plasma. For short-term stability, QC samples were analysed after left at ambient temperature for $4 \mathrm{~h}$. Long-term stability was investigated by analyzing QC samples stored at $-20{ }^{\circ} \mathrm{C}$ for 4 weeks. The QC samples of three freeze-thaw cycles were determined to assess the freeze-thaw stability. To test the post-preparative stability, the processed samples was analysed after stored at $4{ }^{\circ} \mathrm{C}$ in the autosampler vial for $12 \mathrm{~h}$. The stability results 
are presented in Table 2 and were within the accepted variability limits.

Some sample concentrations were upper than the limit of quantification $(1000 \mathrm{ng} / \mathrm{mL})$ of the calibration curve, and then the blank biological matrix was required to dilute these samples. The precision of diluted samples was less than $7.70 \%$, and the accuracy of them was within $-14.46 \%$, which met acceptable criteria of dilution integrity (within $\pm 15 \%$ ). These results indicated that the diluted samples could be determined accurately.

The validated UPLC-MS/MS method was successfully applied for the rats pharmacokinetics of hyperoside after different drug administration routes. The mean plasma concentration-time profile of hyperoside in rats are presented in Fig. 4. The all pharmacokinetic parameters of hyperoside are calculated via Phoenix WinNonlin and are summarized in Table 3.

\section{Discussion}

To obtain good resolution and suitable retention times, different mobile phase was tried such as methanol-water and acetonitrile-water, and added different proportion of acetic acid, ammonium acetate and ammonium formate into water to get a better chromatographic peak shape. Acetonitrile inhibited peak response compared with methanol as mobile phase. In addition, the chromatographic peak was poor after adding ammonium formate and ammonium acetate. Methanol-water (including $0.1 \%$ acetic acid) was used as the mobile phase, the chromatogram is shown in Fig. 3. The chromatographic peak of analytes was

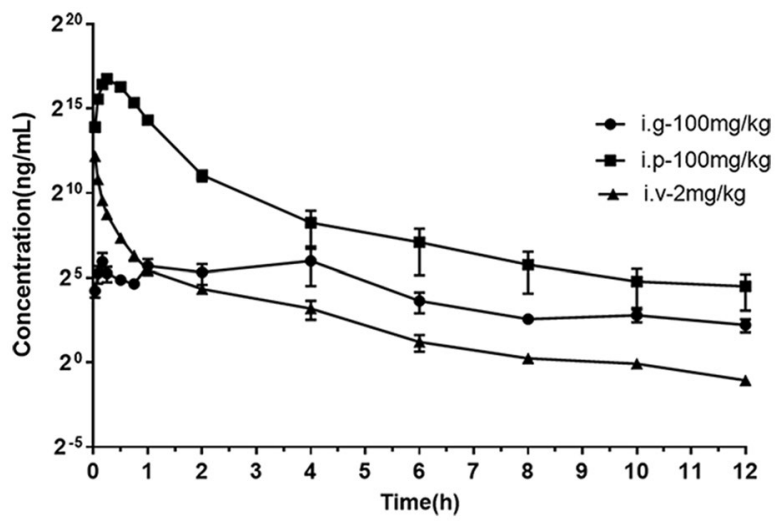

Fig. 4 Mean plasma concentration-time profiles of hyperoside following different administration and does of hyperoside $(n=7)$. $\boldsymbol{\square}$ represents the group of administration via intraperitoneal (i.p); $\boldsymbol{\Delta}$ represents the group of administration via intravenous (i.v); represents the group of administration via intragastric (i.g)

presented with a sharp peak, good symmetry and response intensity. Therefore, methanol (B)-water(A) containing $0.1 \%$ acetic acid was selected as the best mobile phase.

The sample was treated by protein precipitation and liquid-liquid extraction. We found that the samples treated by protein precipitation showed low response of analyte, bifurcation of chromatographic peak. Compared with protein precipitation, liquid-liquid extraction with the organic solvent (ethyl acetate) achieved superior recovery. Therefore, liquid-liquid extraction with ethyl acetate was more suitable as the plasma sample pretreatment method for pharmacokinetic study in rats.
Table 2 Stability of hyperoside under different storage conditions in rat plasma $(n=5)$

\begin{tabular}{lccrl}
\hline Storage conditions & $\begin{array}{l}\text { Spiked concentra- } \\
\text { tion }(\mathrm{ng} / \mathrm{mL})\end{array}$ & $\begin{array}{l}\text { Measured concen- } \\
\text { tration }(\mathrm{ng} / \mathrm{mL})\end{array}$ & RSD (\%) & RE (\%) \\
\hline Short-term (4 h at ambient temperature) & 5 & $5.1 \pm 0.72$ & 14.2 & 2.0 \\
& 20 & $17.7 \pm 0.98$ & 5.5 & -11.5 \\
& 200 & $205.4 \pm 17.74$ & 8.6 & 2.7 \\
Three freeze-thaw cycles & 800 & $791 \pm 47.2$ & 6.0 & -1.1 \\
& 5 & $5.0 \pm 0.58$ & 11.6 & 0 \\
Autosampler for $12 \mathrm{~h}$ & 20 & $18.7 \pm 0.47$ & 2.5 & -6.5 \\
& 200 & $215.0 \pm 6.20$ & 2.9 & 7.5 \\
& 800 & $860.2 \pm 66.71$ & 7.8 & 7.5 \\
& 5 & $5.1 \pm 0.35$ & 6.9 & 1.1 \\
Long-term (4 weeks at $\left.-20{ }^{\circ} \mathrm{C}\right)$ & 20 & $18.6 \pm 0.40$ & 2.2 & -7.0 \\
& 200 & $196.8 \pm 5.45$ & 2.8 & -1.6 \\
& 800 & $840.2 \pm 68.43$ & 8.1 & 5.0 \\
& 5 & $5.1 \pm 0.17$ & 3.5 & 1.1 \\
& 20 & $20.2 \pm 0.48$ & 2.4 & 1.0 \\
& 200 & $192.8 \pm 19.87$ & 10.3 & -3.6 \\
& 800 & $732.5 \pm 44.20$ & 6.0 & -8.4 \\
\hline
\end{tabular}


Table 3 Pharmacokinetic parameters of hyperoside in rats following different administration of hyperoside $(n=7)$

\begin{tabular}{lllll}
\hline Parameter & Units & i.p & i.g & i.v \\
\hline HL_Lambda_z & (h) & 2.14 & 4.12 & 3.28 \\
Tmax & $(\mathrm{h})$ & 0.32 & 1.00 & 0.03 \\
Cmax॰ & $(\mathrm{ng} / \mathrm{mL})$ & $55,310.9$ & 149.0 & 4630.6 \\
AUCall• & $(\mathrm{h} * \mathrm{ng} / \mathrm{mL})$ & $40,535.6$ & 305.1 & 748.3 \\
Vz_obs & $(\mathrm{mL} / \mathrm{kg})$ & 7862.0 & $2,290,414.7$ & $13,835.6$ \\
(Vz_F_obs) & & & & \\
Cl_obs & $(\mathrm{mL} / \mathrm{h} / \mathrm{kg})$ & 2498.5 & $382,994.1$ & 2786.7 \\
(Cl_F_obs) & & & & \\
MRTinf_obs@ & $(\mathrm{h})$ & 0.77 & 5.11 & 0.64 \\
Vss_obs & $(\mathrm{mL} / \mathrm{kg})$ & - & - & 1800 \\
F(\%) & - & 108.3 & 0.8 & - \\
\hline
\end{tabular}

The validation of the method was in accordance with the latest FDA bioanalytical method validation guidance [30]. Figure 4 shows the mean plasma concentration-time profiles following different administration route and dose of hyperoside. The pharmacokinetic parameters of hyperoside were determined using a non-compartmental model. Model analysis were calculated, and are summarized in Table 3. After intravenous administration, hyperoside was quickly absorbed and eliminated in plasma, and hyperoside was detected in plasma within $2 \mathrm{~min}$, which was consistent with previous reports [31]. The data showed that the peak time was relatively short after oral administration of hyperoside. In addition, the $\mathrm{AUC}_{\text {all }}$ for oral administration $(100 \mathrm{mg} / \mathrm{kg})$ and intravenous administration $(2 \mathrm{mg} / \mathrm{kg}$ ) were $305.1 \mathrm{~h} * \mathrm{ng} /$ $\mathrm{mL}$ and $748.3 \mathrm{~h} * \mathrm{ng} / \mathrm{mL}$, respectively. The above results indicated that the bioavailability of oral administration was particularly low. The low bioavailability of flavonoids was the main reason for limiting its clinical application, which was closely related to its intestinal metabolism [32-34], water solubility [35] and efflux transporters [36]. Some of the flavonoids have low solubility in most solvents due to their strong planarity, close arrangement between molecules, small molecular interspace, great molecular attraction and difficulty in solvents. The hydrophobicity of these compounds results in their low bioavailability. On the other hand, the efflux of transporters may be an important cause of low bioavailability. Vaidyanathan et al. [37] found that epicatechin, a flavonoid component in tea, under the action of the multidrugresistance-associated protein2 (MRP2) transporter inhibitor MK-571, the efflux of the prototype and the metabolite was significantly reduced. MRP2 has a significant effect on the bioavailability of epicatechin and other tea flavones. In addition, the first-pass metabolic effect of drugs caused by enzymatic hydrolysis of intestinal flora will also lead to the decrease of bioavailability. Hanske et al. [38] also confirmed that the bioavailability of astragaloside in rats was affected by intestinal flora. The reasons for the low bioavailability of hyperoside are still being investigated. However, when administered intraperitoneally, the bioavailability of hyperoside was relatively high, which was similar to the absorption of intravenous administration. The above data indicated that intraperitoneal injection may be a more suitable method of administration.

\section{Conclusion}

In this paper, we developed a novel UPLC-MS/MS method for the quantification of hyperoside in rat plasma. Compared to the previous methods, the method has the advantages of short analysis time, high sensitivity, good reproducibility and simple sample processing. And this method was fully validated and successfully applied to the pharmacokinetic studies of hyperoside via different administration route in rats. The pharmacokinetic data demonstrated that the plasma levels of intraperitoneal injection were closer to those of intravenous administration than intragastric administration. The study provides a preliminary experimental basis for the choice of hyperoside administration route and dosage form, which is helpful for the later development of hyperoside.

Acknowledgements This work was supported by the National Natural Science Foundation of China $(81673684,81703626,81573690)$ and the "Double first-Class" University project [CPU2018GY33].

\section{Compliance with ethical standards}

Conflict of interest The author declare no conflict of interest.

\section{References}

1. Pan Xinxin Du, Leyue TJ et al (2017) Dynamic changes of flavonoids in Abelmoschus manihot different organs at different growth periods by UPLC-MS/MS. J Chromatogr B AnalytTechnol Biomed Life Sci 1059:21-26

2. On CC, Ching LC, Fung NY (2015) Discrimination between leave of Apocynum venetum and its adulterant, A. pictum based on antioxidant assay and chemical profiles combined with multivariate statistical analysis. Antioxidants 4:359-372

3. Lei W, Yunliang L, Ruimin L et al (2017) An efficient method for the preparative isolation and purification of flavonoids from leaves of Crataegus pinnatifida by HSCCC and pre-HPLC. Molecules 22:767. https://doi.org/10.3390/molecules22050767

4. Li N, Tang H, Wu L et al (2020) Chemical constituents, clinical efficacy and molecular mechanisms of the ethanol extract of Abelmoschus manihot flowers in treatment of kidney diseases. Phytother Res. https://doi.org/10.1002/ptr.6818

5. Yang Z, Xiao-Yan M, Tong Z et al (2019) Apocynum venetum protective effects of against pirarubicin-induced cardiotoxicity. Am J Chin Med 47:1075-1097

6. Zhang LL, Zhang L, Xu J (2020) Chemical composition, antibacterial activity and action mechanism of different extracts from hawthorn (Crataegus pinnatifidaBge.). Sci Rep 10:8876 
7. Ting Fu, Ling W, Xiangnan J et al (2016) Hyperoside induces both autophagy and apoptosis in non-small cell lung cancer cells in vitro. ActaPharmacol Sin 37:505-518

8. Wang X, Fan G, Wei F et al (2019) Hyperoside protects rat ovarian granulosa cells against hydrogen peroxide-induced injury by sonic hedgehog signaling pathway. ChemBiol Interact 310:108759

9. Xiao R, Xiang A, Pang H et al (2017) Hyperoside protects against hypoxia/reoxygenation induced injury in cardiomyocytes by suppressing the Bnip3 expression. Gene 629:86-91

10. Liu R, Xiong Q, Qing S et al (2012) Hyperoside protects cortical neurons from oxygen-glucose deprivation-reperfusion induced injury via nitric oxide signal pathway. Brain Res 1469:164-173

11. Lin Wu, Qing Li, Simeng L et al (2019) Protective effect of hyperoside against renal ischemia-reperfusion injury modulating mitochondrial fission, oxidative stress, and apoptosis. Free Radic Res 53:727-736

12. Huijeong A, Geun-Shik L (2017) Isorhamnetin and hyperoside derived from water dropwort inhibits inflammasome activation. Phytomedicine 24:77-86

13. Ho CJ, DongWook K, Nari Y (2011) Protective effects of hyperoside against carbon tetrachloride-induced liver damage in mice. $\mathrm{J}$ Nat Prod 74:1055-1060

14. Shuo WY, Yan SC, Guo JJ (2019) Antidepressant active ingredients from herbs and nutraceuticals used in TCM: pharmacological mechanisms and prospects for drug discovery. Pharmacol Res 150:104520

15. Zhao J, Tian S, Lu D et al (2020) Systems pharmacological study illustrates the immune regulation, anti-infection, anti-inflammation, and multi-organ protection mechanism of Qing-Fei-Pai-Du decoction in the treatment of COVID-19. Phytomedicine 153315. https://doi.org/10.1016/j.phymed.2020.153315

16. Guo Ai, Zhengming H, Dewen W et al (2012) Pharmacokinetics study of hyperoside in rats. Chin J ExpTradit Med Formulae 19:157-161

17. Li Z, Meng F, Zhang Y et al (2016) Simultaneous quantification of hyperin, reynoutrin and guaijaverin in mice plasma by LC-MS/ MS: application to a pharmacokinetic study. Biomed Chromatogr 30:1124-1130

18. Jianming G, Ping L, Jinao D et al (2012) Application of microdialysis for elucidating the existing form of hyperoside in rat brain: comparison between intragastric and intraperitoneal administration. J Ethnopharmacol 144:664-670

19. Li D, Ruijuan L (2017) Consideration and exploration on pharmacokinetic study of traditional Chinese medicine. Mod Tradit Chin Med Materia Medica World SciTechnol 19:1118-1131

20. Jeong SH, Jang JH, Lee YB (2020) Pharmacokinetic comparison of three different administration routes for topotecan hydrochloride in rats. Pharmaceuticals 13:231. https://doi.org/10.3390/ ph13090231

21. Chen $X$ et al (2011) Zhongguo Zhong yao za zhi $=$ Zhongguo zhongyao zazhi $=$ China J Chin Materia Medica 36(17)2347-2349

22. Sheng CC, Sung TC, Phoung CY et al (2016) Hyperin inhibits nuclear factor kappa B and activates nuclear factor E2-related factor-2 signaling pathways in cisplatin-induced acute kidney injury in mice. IntImmunopharmacol 40:517-523

23. Gong C, Li Z, Qin C et al (2016) Hyperin protects against LPSinduced acute kidney injury by inhibiting TLR4 and NLRP3 signaling pathways. Oncotarget 7:82602-82608
24. Le Z, Siyi He, Fan Y et al (2016) Hyperoside ameliorates glomerulosclerosis in diabetic nephropathy by downregulating miR21. Can J PhysiolPharmacol 94:1249-1256

25. Xiaofei A, Lin Z, Yanggang Y et al (2017) Hyperoside pre-treatment prevents glomerular basement membrane damage in diabetic nephropathy by inhibiting podocyte heparanase expression. Sci Rep 7:6413

26. Xingbin Y, Zhaoxia Li, Yujing Z et al (2013) Development and validation of a highly sensitive LC-ESI-MS/MS method for the determination of hyperoside in beagle dog plasma: application to a pharmacokinetic study. Biomed Chromatogr 27:807-811

27. Xun L, Dong W, Yuan WS et al (2010) LC determination and pharmacokinetic study of hyperoside in rat plasma after intravenous administration. Yakugaku Zasshi 130:873-879

28. Ying YX, Ming LY, Jiang FJ et al (2012) Determination of hyperoside and isoquercitrin in rat plasma by membrane-protected micro-solid-phase extraction with high-performance liquid chromatography. J Sep Sci 35:384-391

29. Aimin T, Ping L, Fei Z (2013) Determination of hyperoside in rat plasma after intravenous administration by UPLC-MS. J Chin PharmacSci 22(6):516-520

30. US Department of Health and Human Services, FDA, Center for Drug Evaluation and Research (CDER). Bioanalytical method validation, guidance for industry (2018). www.fda.gov/downl oads/drugs/guidances/ucm070107.pdf

31. Chengju W, Jiyan C, Wenjie Z et al (2016) Study on pharmacokinetics of hyperoside in rats. J Liaoning Uni TCM 18(10):23-26

32. NagulaRuchika L, Sarika W (2019) Recent advances in topical delivery of flavonoids: a review. J Control Release 296:190-201

33. Peimin D, Lijun Z, Feifei L et al (2015) Triple recycling processes impact systemic and local bioavailability of orally administered flavonoids. AAPS J 17:723-736

34. Kyuichi K, Yasukiyo Y, Junji T (2019) Role of intestinal microbiota in the bioavailability and physiological functions of dietary polyphenols. Molecules 24:370

35. Sun X (2015) Research progress in influence factors of flavonoids compounds bioavailability. China J Tradit Chin Med Pharmacy 30(09):3231-3233

36. Wang YZ, Ao HS, Zheng Y (2009) Advances in studies on effects of interactions between flavonoids with efflux transporter and metabolic enzymes on intestinal absorption of flavonoids. ChinTradit Herb Drugs 40(10):1659-1663

37. Vaidyanathan JB, Walle $\mathrm{T}$ (2001) Transport and metabolism of the tea flavonoid (-)-epicatechin by the human intestinal cell line caco-2. Pharm Res 18(10):1420-1425

38. Hanske L, Loh G, Sczesny S et al (2009) The bioavailability of apigenin-7-glucoside is influenced by human intestinal microbiota in rats. J Nutr 139(6):1095-1102

Publisher's Note Springer Nature remains neutral with regard to jurisdictional claims in published maps and institutional affiliations. 